\title{
Comparación citogenética de Psorophora confinnis (Diptera: Culicidae) en cepas representativas de dos poblaciones colombianas
}

\author{
Clara Hernández ${ }^{1}$, Emelina Reyes ${ }^{1}$, Jaime Rodríguez ${ }^{1}$, Víctor Olano ${ }^{2}$, Alberto Morales ${ }^{2}$, Felio Bello ${ }^{1}$ \\ ${ }^{1}$ Laboratorio de Investigaciones en Entomologia, Biología Celular y Genética, Departamento de Química y \\ Biología, Universidad de La Salle, Bogotá, D.C., Colombia. \\ 2 Laboratorio de Entomología, Instituto Nacional de Salud, Bogotá, D.C., Colombia.
}

\begin{abstract}
Con el propósito de comparar citogenéticamente dos cepas colombianas del mosquito Psorophora confinnis, procedentes de Lorica (Córdoba) y Granada (Meta), se efectuó un estudio de caracterización cariológica. A partir de tejidos cerebrales de larvas de IV estadio, se prepararon los cromosomas mitóticos mediante la técnica de aplastamiento (squash) del tejido. Se efectuaron las mediciones cromosómicas, atendiendo a los siguientes parámetros: brazo corto, brazo largo, relación de brazos, longitud total, longitud relativa, índice centromérico y longitud relativa de los cromosomas. Se compararon los cálculos morfométricos y se aplicaron patrones estandarizados para clasificar los cromosomas en los cariotipos analizados. Se registraron diferencias altamente significativas en la longitud total cromosómica y también en los valores de los brazos cortos y largos de los cromosomas, en las metafases obtenidas de las dos cepas. Teniendo en cuenta los patrones de bandas C y G, se estableció correctamente la ubicación del centrómero y las regiones heterocromáticas asociadas a él e, igualmente, el número de bandas en cada uno de los pares cromosómicos en el cariotipo de las cepas de Lorica y Granada. Los resultados de los datos morfométricos y de los fenotipos de bandas sugieren que $P$ s. confinnis existe en Colombia como un complejo de especies.
\end{abstract}

Palabras clave: Psorophora confinnis, cariotipos, cromosomas, bandas, morfometría cromosómica.

\section{Cytogenetic comparison of Psorophora confinnis (Diptera: Culicidae) in two Colombian representative population strains}

With the purpose of carrying out a cytogenetic comparison of the mosquito Psorophora confinnis from two Colombian strains (Lorica, Córdoba, and Granada, Meta) a karyological characterization study was carried out. Brain tissues from late $4^{\text {th }}$ instar larvae were used to obtain mitotic chromosomes through the squash technique. The chromosomal measurements were made to the following chromosomal characteristics: short arm, long arm, arm ratio, total length, relative length, centromeric index and relative length of the chromosomes. The morphometric data were compared, and standardized patterns were applied in order to classify the chromosomes in the analyzed karyotypes. They registered highly significant differences in the chromosomal total length and also in the values of the short and long arms of the chromosomes in the metaphases of the two strains. The $\mathrm{C}$ and $\mathrm{G}$ banding patterns established the correct position of the centromere and associated heterochromatic regions, as well as the number of bands in each chromosomal pair in the karyotypes from Lorica and Granada strains. The results of morphometric data and the banding phenotypes suggest that $P$ s. confinnis exists in Colombia as a species complex.

Key words: Psorophora confinnis, karyotypes, chromosomes, bands, chromosomal morphometry.

\author{
Correspondencia: \\ F. Bello, Departamento de Química y Biología, Universidad \\ de La Salle, Bogotá, D.C., Colombia \\ fax: 286-8391; e-mail: fbello@atenea.lasalle.edu.co \\ Recibido: 25/04/00; aceptado: 28/07/00
}


Psorophora confinnis (Arribalzaga, 1891) es un mosquito de importancia médico-veterinaria, debido a que es vector eficiente del virus de la encefalitis equina venezolana, tipo epidemoepizoótico (1). Presenta una amplia distribución en las Américas, encontrándose en las regiones noreste, centro y sur de los Estados Unidos, alcanzando también Centroamérica y las Antillas; en Suramérica se encuentra en la mayor parte del continente y llega hasta el norte de Argentina, con excepción de Ecuador, Perú, Chile y Argentina (parte central y sur) (2). En Colombia, se ha registrado la presencia del insecto en los departamentos de Norte de Santander, Santander, Tolima, Boyacá, Meta, Huila, Cundinamarca, Córdoba, La Guajira (3), Arauca (4), Casanare (brote de encefalitis equina venezolana y encefalitis equina del este en Casanare, Informe de evaluación entomológica, Laboratorio de Entomología y Laboratorio Nacional de Referencia, Instituto Nacional de Salud, 1998) y Amazonas (colección de referencia, Laboratorio de Entomología, Subdirección de Epidemiología y Laboratorio Nacional de Referencia, Instituto Nacional de Salud, 1996).

Aunque los mosquitos han sido estudiados extensamente desde el punto de vista de su competencia vectorial, bionomía, taxonomía y control, comparativamente, se han efectuado menos trabajos a nivel citogenético $(5,6)$. Stevens, en 1910 (7), fue uno de los primeros en estudiar el cariotipo de los mosquitos y, desde entonces, se han realizado muchas investigaciones citogenéticas, aunque la mayoría en especies de la subfamilia Anophelinae (8).

En 1961, Breland (9) examinó por primera vez el cariotipo de $P$ s. confinnis y estableció un número diploide de 6 cromosomas. En 1996, en Colombia, Bello et al. (10), a partir de larvas de IV estadio de una colonia de la especie originaria de la población de Ambalema, Tolima, describieron las carac-terísticas de los cromosomas, en los cuales el par 1 fue más corto y metacéntrico, en tanto que los pares 2 y 3 fueron submetacéntricos y de mayor tamaño.

Estudios citogenéticos en la familia Culicidae, que corresponde a los mosquitos, han revelado que el número cromosómico en las especies examinadas, excepto en una, es de seis, de los cuales dos pares son autosómicos y el otro, usualmente más pequeño, pertenece al par sexual. Típicamente los tres pares son metacéntricos; sin embargo, en algunas especies uno o más pares pueden ser submetacéntricos (11-14). La única excepción es Chagasia bathana con un número cromosómico diploide de 8 (15), especie que corresponde a la subfamilia Anophelinae. Sólo en los mosquitos de esta subfamilia, el par sexual es heteromórfico: el cromosoma $X$ es de mayor longitud que el cromosoma $Y(16,17)$.

Una importante característica de los cromosomas de mosquitos, la cual es compartida por otros dípteros, es la casi permanente 'sinapsis' de los cromosomas homólogos, fenómeno llamado 'pareamiento somático' (18), que se identifica como una estrecha asociación de estos cromosomas, no sólo a nivel de sus centrómeros sino también a través de sus longitudes durante algunas etapas de la división mitótica (profase y prometafase), dando la aparente visión de un número diploide de 3 cromosomas. En células germinativas de mosquitos de la subfamilia Culicinae, el fenómeno de pareamiento somático repercute durante la meiosis, ocasionando ausencia de los clásicos estadios de leptoteno y cigoteno durante la profase I $(19,20)$.

Las técnicas de bandas cromosómicas que fueron originalmente desarrolladas en mamíferos, con particular énfasis en cromosomas humanos (2128), han sido también adaptadas para los análisis citogenéticos de insectos; no obstante, existen pocas especies de mosquitos cuyos cariotipos se hayan estudiado utilizando estos marcadores.

Tal vez donde mayor aplicación ha tenido una de estas técnicas, bandas $\mathrm{C}$, ha sido en algunas especies del género Aedes (18,29,30), en cuyos cromosomas, además de poder identificar con este método el centrómero y la región de la heterocromatina constitutiva asociada a él, también ha sido posible visualizar una banda intercalar del locus sexual en los cromosomas homólogos del par 1, lo cual ha permitido, igualmente y por las características de esta banda de heterocromatina facultativa, diferenciar las especies estudiadas e inferir sus relaciones filogenéticas (29). 
El conocimiento genético (citogenética, caracterización genética y molecular) de las especies de insectos vectores es necesario para explicar y comprender las directrices evolutivas en sentido biológico, fisiológico y cromosómico con su correspondiente grado de diferenciación fenotípica. Además, los métodos de control y su aplicación con éxito dependen principalmente del análisis de la estructura genética de las poblaciones naturales.

Con el propósito de comparar las características citogenéticas de dos cepas del mosquito Ps. confinnis, originarias de las poblaciones colombianas de Lorica (Córdoba) y Granada (Meta), se efectuó un estudio morfométrico de los cromosomas con patrones de bandas $\mathrm{C}$ y $\mathrm{G}$.

\section{Materiales y métodos}

\section{Muestras biológicas}

Se utilizaron larvas de IV estadio de Ps. confinnis, las cuales fueron tomadas de dos colonias iniciadas a partir de formas inmaduras y de adultos, recolectadas en áreas cercanas a los municipios de Lorica (Córdoba) (32) y Granada (Meta) (Laboratorio de Entomología, Universidad de La Salle). Las capturas para la iniciación de la primera colonia se efectuaron los días 14 y 15 de febrero de 1998 y hasta la fecha se han obtenido 32 generaciones. La cepa Granada se comenzó el 6 de noviembre de 1997 y se han registrado 36 generaciones continuas. Ambas colonias se mantienen a una temperatura promedio de $27^{\circ} \mathrm{C}$, $85 \%$ de humedad y 12 horas de luz.

\section{Preparación de cromosomas mitóticos}

Las larvas de IV estadio de cada una de las colonias fueron procesadas individualmente de acuerdo con los procedimientos descritos por French et al. (33) y Marchi et al. (34), con algunas modificaciones. Las larvas se colocaron en una solución de colchicina al $0,1 \%$ durante 5 horas, previo al proceso de disección, el cual se efectuó en presencia de una solución de citrato de sodio al $1 \%$; con la ayuda de una aguja entomológica, se separó el tórax de la cabeza y se sacó el cerebro por la parte posterior de la cápsula cefálica, observando el procedimiento con el estereoscopio. El cerebro constituido por dos lóbulos fue trasladado a una laminilla a la que previamente se le colocó una gota de fijador, ácido acético al $45 \%$; luego se adicionó a la preparación una gota de orceína lactoacética y se dejó actuar este colorante durante 45 minutos. Se colocó una lámina sobre el micropreparado y se procedió a efectuar una presión fuerte del dedo pulgar sobre la laminilla (squash). Las mejores metafases se fotografiaron, utilizando un microscopio BX-60 con sistema microfotográfico PM-PBK-3, marca Olympus. Las mediciones estándar para cada uno de los cromosomas se realizaron directamente sobre las fotos de los cariotipos, teniendo en cuenta para los cálculos de la longitud en micras de los cromosomas, los aumentos dados por el microscopio y por la ampliadora (paso de negativo a papel), que en total fueron $3.000 \mathrm{X}$.

\section{Bandas C}

Las láminas sin tinción, obtenidas a partir de la técnica de squash y sometidas a un tiempo de maduración de 10 dias, fueron sumergidas en una solución de ácido clorhídrico $0,2 \mathrm{~N}$ durante 30 minutos; luego de previos lavados con agua destilada, se trataron con hidróxido de bario al $5 \%$ a $53^{\circ} \mathrm{C}$ por un tiempo de 15 segundos $y$, antes de ser coloreadas con Giemsa al $2 \%$, se colocaron durante una hora en solución salina doblemente citratada a una temperatura de $60^{\circ} \mathrm{C}(18,29,30)$.

\section{Bandas G}

El tiempo de maduración para las láminas sin tinción fue de 25 días, al término de los cuales se incubaron en una solución doblemente citratada a $60{ }^{\circ} \mathrm{C}$ durante 45 minutos; previo enjuague, se sumergieron en una solución de tripsina al $0,1 \%$ a $10^{\circ} \mathrm{C}$ durante 40 segundos, al cabo del cual se detuvo su acción con etanol. La coloración se efectuó con Giemsa al $2 \%$ durante 60 minutos $(34,35)$.

\section{Morfometría cromosómica}

Se seleccionaron 20 metafases de cada una de las cepas, las cuales se obtuvieron individualmente a partir de tejidos de cerebro, correspondientes a larvas diferentes, en las cuales se efectuaron las siguientes mediciones directas: brazo corto $(p)$, brazo largo (q) y longitud total (LT). También se calcularon las indirectas: relación de brazos $(q / p$ 
y $p / q)$, longitud relativa (LR), que corresponde a la longitud total de cada cromosoma sobre la longitud total del genoma, índice centromérico (IC), que se encontró midiendo la relación del brazo corto de cada cromosoma sobre la longitud total del mismo y, finalmente, la longitud relativa de los cromosomas (LRC) se registró después de relacionar la longitud total de cada cromosoma sobre la longitud del cromosoma más pequeño del genoma.

Para el análisis de los datos, correspondientes a las mediciones de $p, q$ y LT, se utilizó la prueba estadística de la t de Student (36), mediante la cual se pudo determinar la diferencia de los valores obtenidos en ambas poblaciones.

Para la clasificación de los cromosomas, se utilizaron los datos directos de las mediciones de los brazos, teniendo en cuenta la posición del centrómero; también, se emplearon los parámetros establecidos por Zimmerman, Levan y Ospina De Dulce (37-39).

\section{Resultados}

\section{Número cromosómico}

Las metafases mitóticas obtenidas de tejidos cerebrales, correspondientes al mosquito PS. confinnis en las dos cepas, exhibieron tres pares de cromosomas, número diploide $2 n=6$; el número fundamental fue de 12. Estos pares cromosómicos se numeraron en orden ascendente de tamaño como par 1, par 2 y par 3 (figuras 1 y 2 ).

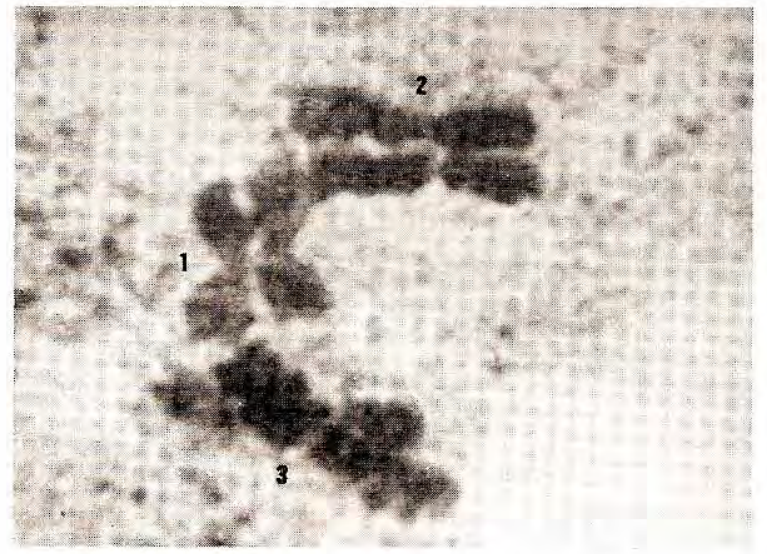

Figura 1. Cariotipo de Ps. confinnis, cepa Lorica.

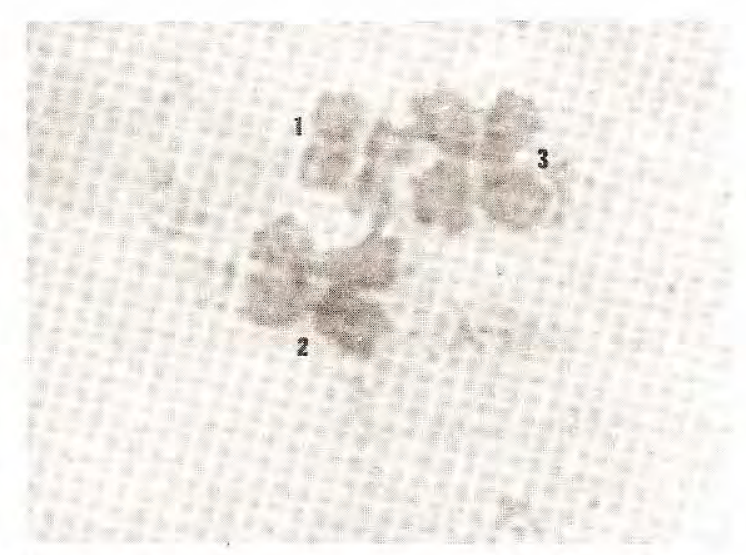

Figura 2. Cariotipo de Ps. confinnis, cepa Granada.

\section{Longitudes cromosómicas}

En el cuadro 1 se muestran los promedios de las longitudes cromosómicas de Ps. confinnis, correspondientes a las cepas de Lorica y Granada.

En la figura 3 se observan, en forma comparativa, las longitudes cromosómicas del cariotipo de la especie en ambas cepas, atendiendo a los datos de longitud total. En la figura 4, se observan, comparativamente, los idiogramas de la longitud total del cariotipo del mosquito en las dos poblaciones.

\section{Análisis estadístico}

En los cuadros 2 y 3 , se muestra la estadística descriptiva para las longitudes seleccionadas de los cromosomas, correspondientes a los cariotipos de las cepas Lorica y Granada. Se presentaron porcentajes de variación relativamente bajos para todas las mediciones en las dos poblaciones, como lo demuestra el coeficiente de variación que fue determinado en 14 y $27 \%$, lo que nos permite tener una alta confiabilidad en las diferencias obtenidas en las dos cepas, así como en las inferencias que se realizaron a partir de los datos estadísticos calculados.

En el cuadro 4, se observan los valores de la t de Student aplicados a la comparación de las longitudes cromosómicas ( $p, q$ y LT), pertenecientes al mosquito en las poblaciones relacionadas. Se presentaron diferencias altamente significativas en los valores de las longitudes cromosómicas analizadas entre las dos cepas. 
Cuadro 1. Promedio de las longitudes cromosómicas de Ps. confinnis en los cariotipos de las cepas comparadas.

\begin{tabular}{|c|c|c|c|c|c|c|c|c|c|c|c|c|c|c|c|c|c|}
\hline Par & & $p$ & q & 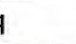 & & LT & & $R$ & $q /$ & $p$ & $\mathrm{p} /$ & & IC & C & & $\mathrm{RC}$ & $\mathrm{n}$ \\
\hline 1 & 3,06 & 1,40 & 3,06 & 1,40 & 6,12 & 2,80 & 0,24 & 0,24 & 1,00 & 1,00 & 1,00 & 1,00 & 0,50 & 0,50 & 1,00 & 1,00 & 20 \\
\hline 2 & 3,93 & 1,80 & 4,86 & 2,06 & 8,79 & 3,86 & 0,35 & 0,33 & 1,23 & 1.14 & 0,80 & 0,87 & 0,44 & 0,46 & 1,43 & 1,37 & 20 \\
\hline 3 & 4,63 & 2,20 & 5,53 & 2,66 & 9,76 & 4,86 & 0,39 & 0,42 & 1,10 & 1.20 & 0,90 & 0,82 & 0,47 & 0,45 & 1,49 & 1,73 & 20 \\
\hline
\end{tabular}

p: brazo corto, q: brazo largo, LT: longitud total, LR: longitud relativa, IC: índice centromérico, LRC: longitud relativa de los cromosomas

Longitud en micras; * $\mathrm{n}$ : número de metafases

Cepa Lorica

Cepa Granada

Cuadro 2. Estadistica descriptiva para la longitud de los cromosomas de PS. confinnis en la cepa Lorica.

\begin{tabular}{|c|c|c|c|c|c|c|c|c|c|}
\hline \multicolumn{2}{|r|}{ Cromosoma } & $\begin{array}{c}\text { Brazos } \\
\text { cromosómicos }\end{array}$ & Promedio & $\begin{array}{c}\text { Desviación } \\
\text { estándar }\end{array}$ & \multicolumn{2}{|c|}{$\begin{array}{l}\text { Valor observado } \\
\text { mínimo máximo }\end{array}$} & $\begin{array}{l}\text { Interval } \\
\text { lím. inf. }\end{array}$ & $\begin{array}{l}\text { de } 95 \% \\
\text { lim. sup. }\end{array}$ & $\begin{array}{c}\text { Coeficiente } \\
\text { de } \\
\text { variación }\end{array}$ \\
\hline \multirow{2}{*}{\multicolumn{2}{|c|}{ Par uno }} & $\mathrm{p} 1$ & 0,86 & 0,17 & 0,60 & 1,25 & 1,64 & 0,96 & 20,32 \\
\hline & & q1 & 0,86 & 0,17 & 0,60 & 1,25 & 0,76 & 0,96 & 20,32 \\
\hline L & & Longitud total & 1,82 & 0,32 & 1,40 & 2,60 & 0,76 & 2,00 & 17,74 \\
\hline 0 & & & & & & & & & \\
\hline $\mathrm{R}$ & \multirow[t]{3}{*}{ Par dos } & p2 & 1,10 & 0,23 & 0,80 & 1,80 & 0,97 & 1,23 & 21,26 \\
\hline 1 & & q2 & 1,36 & 0,20 & 1,20 & 1,80 & 1,25 & 1,47 & 14,41 \\
\hline C & & Longitud total & 2,46 & 0,46 & 1,70 & 3,40 & 2,20 & 2,72 & 18,76 \\
\hline \multirow{3}{*}{\multicolumn{2}{|c|}{ Par tres }} & p3 & 1,29 & 0,23 & 1,00 & 1,90 & 1,16 & 1,42 & 17,74 \\
\hline & & $q^{3}$ & 1,43 & 0,24 & 1,20 & 2,10 & 1,29 & 1,57 & 17,03 \\
\hline & & Longitud total & 2,74 & 0,44 & 2,20 & 3,70 & 2,49 & 2,99 & 15,96 \\
\hline
\end{tabular}

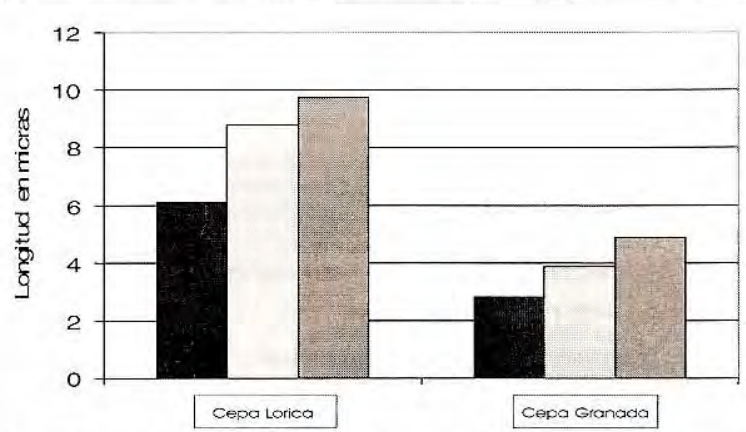

Figura 3. Histograma comparativo de la longitud total cromosómica en el cariotipo de Ps. confinnis, correspondiente a las dos cepas.

\section{Clasificación de los cromosomas}

Con los datos directos y teniendo en cuenta la posición del centrómero, los cromosomas en ambas cepas se clasificaron así: par 1 como metacéntrico y los pares 2 y 3 ligeramente submetacéntricos. De acuerdo con los parámetros establecidos por los tres autores, los cromosomas del cariotipo de Ps. confinnis en las dos cepas aparecen clasificados en los cuadros 5 y 6 . Sólo el par 3, en ambas poblaciones, presenta discrepancia en cuanto a su clasificación.

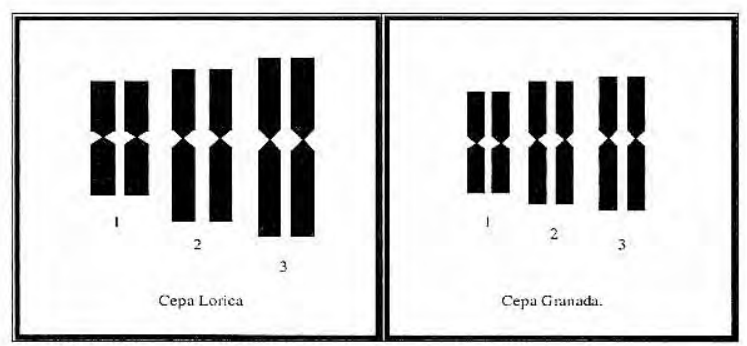

Figura 4. Comparación del cariotipo de Ps. confinnis en ambas cepas con base en sus idiogramas.

\section{Bandas C}

Mediante esta técnica se pudo identificar correctamente el centrómero y la región de heterocromatina constitutiva asociada con él, presente en cada uno de los pares cromosómicos de los cariotipos en las dos cepas (figuras 5 y 6 ). La distribución de la heterocromatina constitutiva está restringida a las regiones pericentroméricas, sin encontrarse bandas intercalares en ninguna de las poblaciones. Aparentemente, hay mayor cantidad en la cepa Lorica. 
Cuadro 3. Estadística descriptiva para la longitud de los cromosomas de Ps. confinnis en la cepa Granada.

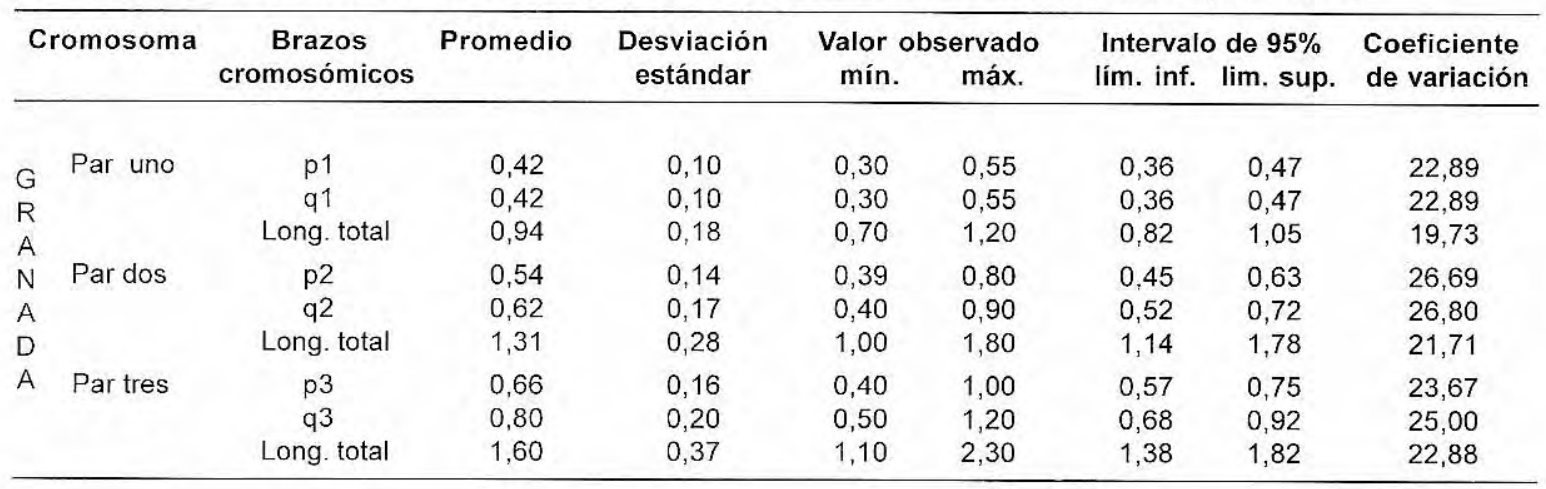

Cuadro 4. Valores de la t de Student para la comparación de las longitudes cromosómicas en los cariotipos de las cepas Lorica y Granada.

\begin{tabular}{lccc}
\hline Cromosoma & $\mathbf{P}$ & $\mathbf{q}$ & Longitud total \\
\hline Par 1 & $7,85^{\star *}$ & $7,85^{* *}$ & $8,35^{* *}$ \\
Par 2 & $7,12^{* *}$ & $9,71^{* *}$ & $7,39^{\star *}$ \\
Par 3 & $7,87^{* *}$ & $6,79^{* *}$ & $6,73^{* *}$ \\
\hline
\end{tabular}

** Diferencias altamente significativas $p<=0,01$

* Diferencias significativas $0,01<p<=0,05$

$\mathrm{SN}$ : sin diferencias significativas

Cuadro 5. Clasificación cromosómica de Ps. confinnis para la cepa Lorica.

\begin{tabular}{lccc}
\hline Cr.* & Levan & Zimmerman & de Dulce \\
\hline Par 1 & Metacéntrico & Metacéntrico & Metacéntrico \\
Par 2 & Metacéntrico & Submetacéntrico & Metacéntrico \\
Par 3 & Submetacéntrico & Submetacéntrico & Metacéntrico \\
\hline
\end{tabular}

${ }^{*} \mathrm{Cr}$ : cromosomas

Cuadro 6. Clasificación cromosómica de Ps. confinnis para la cepa Granada.

\begin{tabular}{lccc}
\hline Cr.* $^{*}$ & Levan & Zimmerman & de Dulce \\
\hline Par 1 & Metacéntrico & Metacéntrico & Metacéntrico \\
Par 2 & Metacéntrico & Submetacéntrico & Metacéntrico \\
Par 3 & Metacéntrico & Submetacéntrico & Metacéntrico \\
\hline
\end{tabular}

${ }^{*} \mathrm{Cr}$ : cromosomas

\section{Bandas G}

El examen de los cariotipos desarrollados con esta técnica, mostró claros patrones de bandas en las dos poblaciones, los cuales se observan en las figuras 7 y 8 . El número de bandas en los cromosomas del cariotipo de la especie en la cepa Lorica fueron los siguientes: par 1 de 7 a 8 , par 2 de 10 a 11 y par 3 de 11 a 12 . En la cepa Granada,

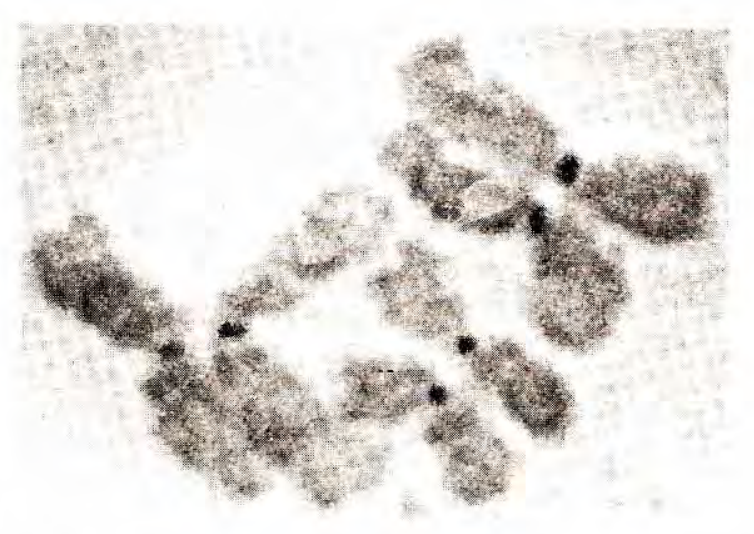

Figura 5. Complemento cromosómico de Ps. confinnis, cepa Lorica, mostrando bandas centroméricas, método de bandas $\mathrm{C}$ y tinción con Giemsa.

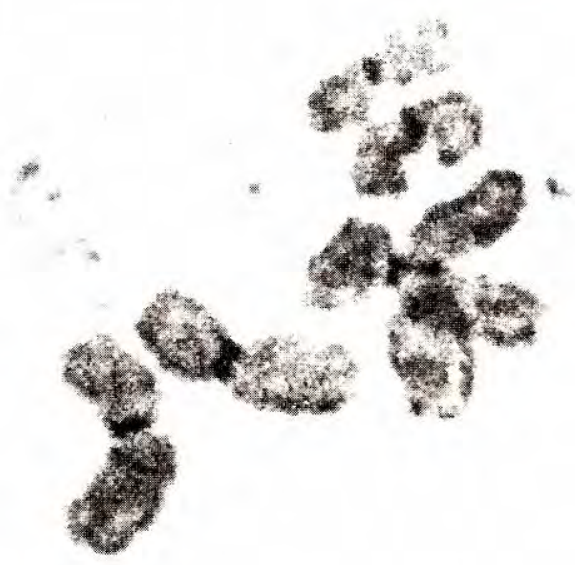

Figura 6. Complemento cromosómico de Ps. confinnis, cepa Granada, mostrando bandas centroméricas, método de bandas $C$ y tinción con Giemsa. 


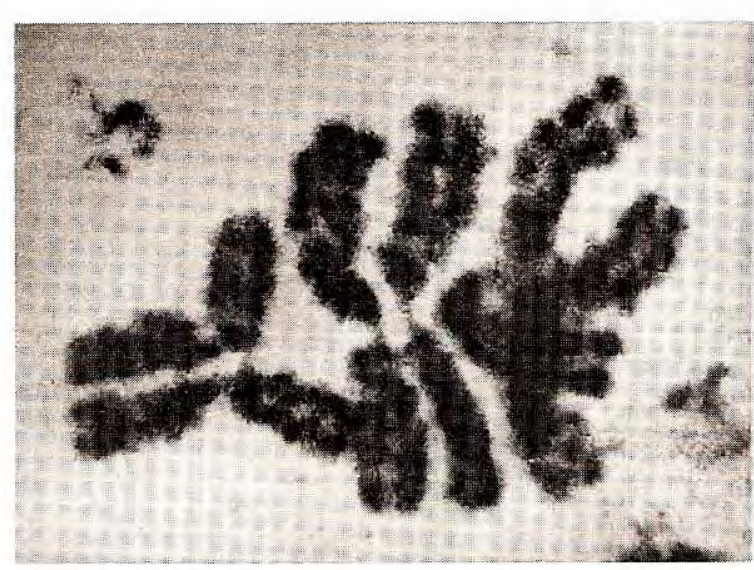

Figura 7. Patrones de bandas $\mathrm{G}$ en el cariotipo de PS. confinnis, cepa Lorica.

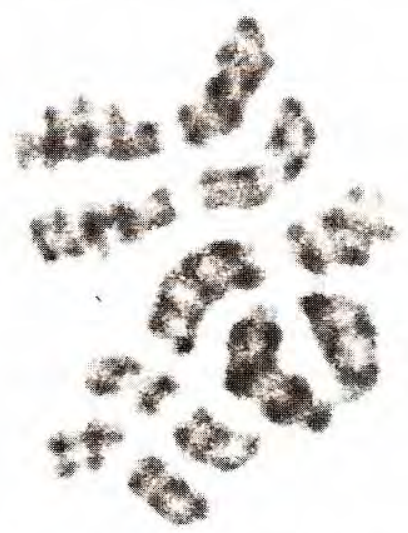

Figura 8. Patrones de bandas $\mathrm{G}$ en el cariotipo de PS. confinnis, cepa Granada.

se registraron de 5 a 6 bandas en el par 1 , de 7 a 8 bandas en el par 2 y de 9 a 10 bandas en el par 3. En la figura 9 se muestra el histograma comparativo de bandas $\mathrm{G}$ en las dos cepas.

Para los análisis de bandas, se seleccionaron 10 metafases de cada cepa, las que a su vez correspondieron a igual número de preparaciones, obtenidas de diferentes larvas.

\section{Discusión}

El número cromosómico diploide de seis, en las dos cepas estudiadas, coincidió con el señalado para la especie por Breland (9). También este número fue igual al reportado por Bello et al. (10), con muestras del mosquito tomadas de una colonia originaria de Ambalema, Tolima.

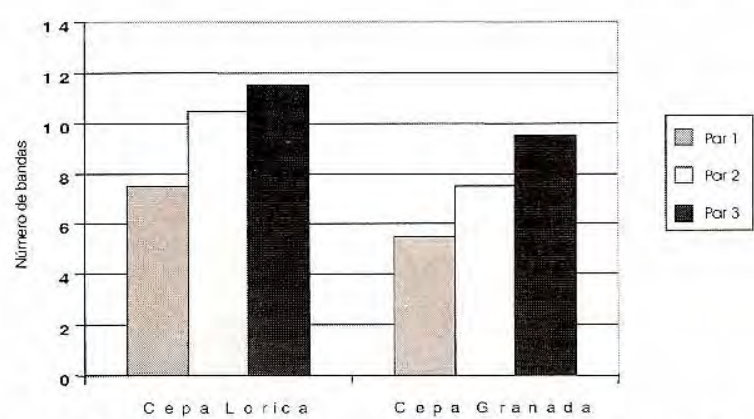

Figura 9. Histograma comparativo de los promedios de bandas $\mathrm{G}$ en el cariotipo de PS. confinnis, correspondiente a las dos cepas.

Al comparar la longitud cromosómica total de PS. confinnis en las dos cepas, observamos que los promedios de las mediciones en la población Lorica son de mayor tamaño; también resultaron diferencias altamente significativas al aplicar la t de Student (cuadro 4) con base en los datos estadísticos obtenidos de los valores correspondientes a brazo corto, brazo largo y longitud total de los cromosomas. De forma análoga, al comparar el patrón de bandas $C$ en los cariotipos de las cepas de Lorica y Granada, se registraron marcadas diferencias en la longitud del centrómero y las regiones heterocromáticas asociadas a él en los respectivos pares cromosómicos, basadas en una mayor cantidad y distribución de dichos materiales en la primera cepa. Así mismo, en los resultados obtenidos con la técnica de bandas $G$ aplicada a las metafases cromosómicas en las dos poblaciones del mosquito, se observaron diferencias relativas en el número de bandas, siendo mayor en la cepa Lorica, lo que en cierta forma corrobora las diferencias establecidas en las longitudes totales. En conjunto, el análisis de los resultados sugiere que, probablemente, las poblaciones de Ps. confinnis en Colombia pueden existir en forma de un complejo de especies. Un estudio preliminar (Lanzaro, 1997, datos no publicados, comunicación personal) reveló la presencia de dos variedades diferentes de la especie en los Estados Unidos y México, columbiae y toltecum. Ps. confinnis propiamente dicha estaría distribuida más hacia el sur del continente americano; sin embargo, en Colombia es posible que las tres variedades puedan existir y sus probables rangos de distribución serían: 
hacia el norte, Ps. toltecum; en la región central, Ps. columbiae, y hacia el sur, Ps. confinnis, propiamente dicha.

Los valores promedio de las longitudes cromosómicas de Ps. confinnis en las cepas estudiadas, son relativamente mayores comparados con los resultados obtenidos en las medidas cromosómicas en otras especies $(40,41)$, lo cual indica diferencias en la cantidad de ADN nuclear entre los insectos analizados. Rao y Rai encontraron una buena correlación entre la longitud cromosómica y el contenido de ADN nuclear de 36 especies de mosquitos (42); observaron que la longitud total de los cromosomas varía de $8,4 \mathrm{m \mu}$ en Anopheles quadrimaculatus a $38,3 \mathrm{m \mu}$ en Aedes zoosophus, correspondiendo, a su vez, a los valores mínimo y máximo de 0,24 pg y 1,90 pg, respectivamente, en núcleos haploides de las células de testículos de pupas, los cuales fueron determinados por citofotometría Feulgen.

Con base en las mediciones directas y la posición del centrómero en los pares cromosómicos de los cariotipos, en ambas cepas analizadas, se pudo establecer que el par 1 fue metacéntrico y de menor longitud, mientras que los pares 2 y 3 fueron ligeramente submetacéntricos y de mayor tamaño; no obstante, con los criterios utilizados por los tres autores para clasificar los cromosomas, observamos que sólo Zimmerman coincidió con la anterior clasificación. Levan y Ospina de Dulce, en términos casi semejantes, clasifican los cromosomas del cariotipo como metacéntricos; sin embargo, no se puede esperar que haya una coincidencia total entre los autores antes señalados, ya que los rangos y parámetros establecidos por cada uno de ellos para clasificar los cromosomas en el cariotipo son distintos.

En el cariotipo de mosquitos de las subfamilias Culicinae y Toxorhynchitinae, el par 1 es homomórfico y contiene el locus sexual, mientras que en los anofelinos, el par sexual es heteromórfico $(X, Y)$, en donde $X$, en la mayoría de los casos, es submetacéntrico y de mayor longitud, y $Y$ puede ser submetacéntrico, acrocéntrico e irregular $(16,17)$. Con la técnica de bandas $C$, ha sido posible la identificación del locus sexual en algunas especies del género Aedes (31); sin embargo, no ocurrió lo mismo en el presente trabajo con Ps. confinnis, debido a que no hubo coloración diferencial en los brazos del cromosoma 1 al aplicar la mencionada técnica. Motara y Rai mostraron que especies estrechamente relacionadas, con más o menos similares cariotipos (Ae. aegypti y Ae. mascarensis), se separan con la técnica de bandas $C$, originando la presencia de una banda intercalar en uno de los cromosomas del par sexual (30). En este caso, se identificó como el locus sexual femenino en la primera especie, en tanto que, para $A e$. mascarensis, dicha banda no estuvo presente y sólo hubo la coloración de las bandas centroméricas en cada uno de los cromosomas. Motara y Rai extendieron sus estudios de bandas $C$ a nueve especies, también del género Aedes, determinando -a través del análisis correspondiente- distintos patrones de bandas, lo cual permitió una clara diferenciación entre los organismos estudiados (29). La presencia de bandas intercalares puede ser consecuencia de cambios evolutivos en la cantidad y la distribución de la heterocromatina en los cromosomas de las especies correspondientes a este género.

No obstante, los resultados del presente trabajo, en el que se han podido establecer diferencias citogenéticas de Ps. confinnis en Colombia a partir de muestras tomadas de colonias originarias de dos poblaciones, es necesario continuar trabajando con otros marcadores, principalmente a nivel molecular, en estudios genéticos de las poblaciones naturales del mosquito en diferentes sitios del país, debido a que una correcta y precisa diferenciación de la especie es determinante en la definición de particulares características de competencia y capacidad vectorial que nos permitan entender el papel del vector en la epidemiología de la enfermedad.

\section{Agradecimientos}

Al licenciado Jesús Escovar, auxiliar del Laboratorio de Investigaciones de Entomología de la Universidad de La Salle por el suministro del material biológico.

A Colciencias (proyecto 1243-05-278-97), la Universidad de La Salle y el Instituto Nacional de Salud por la financiación del presente trabajo. 


\section{Referencias}

1. PAHO. Panamerican Sanitary Bureau. Venezuelan Encephalitis. Scientific Publication. No. 243. Washington D.C.; 1972. p.157-61.

2. Forattini OP. Entomología médica. Sao Paulo: Universidade de Sao Paulo, Brasil; 1965. p.422-8.

3. Olano VA, Morales A. Colonización de una cepa de Psorohora (Grabhamia) confinnis Arribalzaga, 1891, en Colombia. Biomédica 1981;1:12-5.

4. González C. Mosquitos de Caño Limón, Arauca (Diptera: Culicidae) (tesis). Bogotá, D.C., Colombia: Universidad Nacional de Colombia; 1995.

5. Clements AN. The biolngy of mosquitoes. Development, nutrition and reproduction. London: Chapman \& Hall; 1992. p.1-44.

6. Coker WZ. Formal genetics and cytogenetics of Aedes aegypti. Bull World Health Org 1967;36:555-8.

7. Stevens NM. The chromosomes in the germ cells of Culex. J Exp Zool 1910;8:205-25.

8. Knudson DL, Zheng L, Gordon SW, Brown SE, Kafatos FC. Genome organization. In: Beaty BJ, Marquardt WC, editors. The biology of disease vectors. Boulder, CO: University Press of Colorado; 1996. p.175214.

9. Breland OP. Studies on the chromosomes of mosquitoes. Ann Entomol Soc Am 1961;54:360-75.

10. Bello FJ, Neira M, Olano VA, Ferro C. Estudio cariológico de Psorophora confinnis (Diptera: Culicidae). Diógenes 1996;3:167-76.

11. Rai KS. A comparative study of mosquito karyotypes. Ann Entomol Soc Am 1963;56:160-70.

12. Rai KS. Further observations on the somatic chromosome cytology of some mosquitoes (Diptera: Culicidae). Ann Entomol Soc Am 1966;59:242-6.

13. Kitzmiller JB. Genetics, cytogenetics and evolution of mosquitoes. Adv Genet 1976;18:315-433.

14. Rai KS, Pashley DP, Munstermann LE. Genetics of speciation in aedine mosquitoes. In: Steiner WMN, Tabachnick KS, Rai KS, Narang S, editors. Recent developments in genetics of insect disease vectors. Champaign, III: Stipes Publishers; 1982. p.84-129.

15. Kreutzer RD. A mosquito with eigth chromosomes: Chagasia bathana Dyar. Mosquito News 1978;38:554-8.

16. Kitzmiller JB. Mosquito cytogenetics. In: Wright JW, Pal $R$, editors. Genetics of insect vectors of disease. Amsterdam: Elsevier 1967. p.133-50.

17. Heckel DG. Comparative genetic linkage mapping in insect. Ann Rev Entomol 1993;38:381-408.

18. Newton ME, Southern DI, Wood RJ. $X$ and $Y$ chromosomes of Aedes aegypti (L) distinguished by Giemsa C-banding. Chromosoma 1974;49:41-9.
19. Breland OP, Gassner G, Riemann JG. Studies of meiosis in the male of the mosquito Culiseta inornata. Am Ent Soc America 1964;57:472-9.

20. Mescher SAL, Rai KS. Spermatogenesis in Aedes aegypti. Mosquito News 1966;26:45-51.

21. Cassperson T, Zech L, Johansson C. Analysis of human metaphase chromosome set by aid of DNAbinding fluorescent agents. Exp Cell Res 1970;62:4902.

22. Drets ME, Shaw MW. Specific banding patterns of human chromosome. Proc Nat Acad Sci USA 1971; 68:2073-7.

23. Patil SR, Merrick S, Lubs HA. Identification of each human chromosome with a modified Giemsa stain. Science 1971;173:821-2.

24. SchnedI W. Banding pattern of human chromosomes. Nature 1971;233:93-4

25. Summer AT, Evans HJ, Buckland RA. New technique for distinguishing between human chromosome. Nature 1971:232:31-2.

26. Seabright $\mathbf{M}$. A rapid banding technique for human chromosomes. Lancet 1971;2:971-2.

27. Wang HC, Federoff S. Banding in human chromosomes treated with trypsin. Nature 1972;235:52-4.

28. Caspersson T, Zech L, Johansson C. Analysis of human metaphase chromosome set by aid of DNAbanding fluorescent agent. Exp Cell Res 1970;62:490-2.

29. Motara MA, Rai KS. Giemsa C-banding patterns in Aedes (Stegomyia) mosquitoes. Chromosoma 1978;70: $51-8$.

30. Motara MA, Rai KS. Chromosomal differentiation in two species of Aedes and their hybrids revealed by Giemsa C-banding. Chromosoma 1977;64:125-32.

31. Rai KS. Evolutionary cytogenetics of Aedine mosquitoes. Genetica 1980;52/53:281-90.

32. Alarcón J, Olano V, Morales A, Rodríguez J, Bello F. Colonización de Psorophora confinnis (Diptera: Culicidae), cepa Lorica-Córdoba, Colombia. Diógenes 1999;5:181-91.

33. French WL, Baker RH, Kitzmiller JB. Preparation of mosquito chromosomes. Mosquito News 1962;22:37783

34. Marchi A, Mezzanote R, Ferrucci L. Characterization of the metaphase chromosomes in Anopheles stephensi (Liston, 1901) by $Q, G$ and $C$ banding. Cytologia 1980; 45: $549-53$

35. Marchi A, Rai KS. Chromosome banding homologies in three species of Aedes (Stegomyia). Can J Genet Cytol 1986; 28:198-202.

36. Dowinie NM, Health RW. Métodos estadísticos. Barcelona, España: Editorial Harla; 1973. p.365-70. 
37. Levan A, Fredga K, Sanaberg A. Nomenclature for centromeric positions on chromosomes. Hereditas 1964 52:201.

38. Zimmerman EG, Shivonen G. Chromosomal banding pattern and idiogram of the cotton rat Sigmodon arizonae (Rodentia: Muridae). Chromosoma 1973;41:85-91.

39. García BE. Estudio citotaxonómico de los peces Nematobrycon palmeri y Nematobrycon iacotei (tesis). Bogotá: Pontificia Universidad Javeriana; 1980.
40. Durán F. Establecimiento y mantenimiento de una colonia de Aedes taeniorhynchus (Diptera: Culicidae) cepa Cartagena y su estudio cariológico en condiciones in vivo (tesis). Bogotá, D.C.: Universidad De La Salle; 1996.

41. Asman SM. Cytogenetic observations in Culex tarsalis: mitosis and meiosis. J Med Entomol 1974;11:375-82.

42. Rao PN, Rai KS. Genome evolution in the mosquitoes and other closely related members of superfamily Culicoidea. Hereditas 1990;113:139-44. 